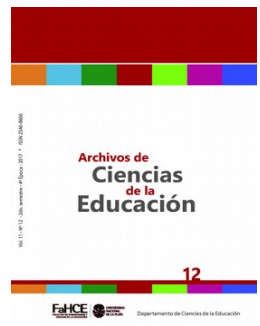

Archivos de Ciencias de la Educación, Vol 11, n 12, diciembre 2017, e028. ISSN 2346-8866 Universidad Nacional de La Plata.

Facultad de Humanidades y Ciencias de la Educación.

Departamento de Ciencias de la Educación.

\title{
Introducción: pasado y presente del aprendizaje y la enseñanza significativos
}

\author{
Analía Mirta Palacios* y María Alejandra Pedragosa* \\ * Universidad Nacional de La Plata, Argentina | apalacios@fahce.unlp.edu.ar; \\ apedragosa@fahce.unlp.edu.ar
}

Y debido a que en toda la Galaxia no habían

encontrado nada más precioso que la Mente, alentaron por doquiera su amanecer (Clarke, 1968)

En la novela "Una odisea al espacio" de Arthur Clarke, publicada en 1968, corría el año 2001. Allí seres de una antigua civilización buscaban el origen de una vida inteligente y se encontraron con "la mente". Con esta ficción, Hampson y Morris (1996) ilustran cómo las investigaciones cognitivas de la época exploraron el funcionamiento de la mente y arrojaron luz sobre distintos aspectos cognoscitivos del proceso de enseñanza y aprendizaje. En especial, en los países de habla anglosajona -Estados Unidos e Inglaterra-, se gestaron los estudios pioneros acerca de cómo la memoria procesa y representa la información y cómo puede ser emplea do el conocimiento para llevar a cabo distintas tareas de la vida inteligente, tales como: recordar, hablar, realizar actividades específicas, resolver problemas y razonar. Mientras tanto, en Ginebra, se fortalecían los estudios sobre el desarrollo de la inteligencia en la perspectiva de la epistemología genética de Piaget. Así, de uno y otro lado del Atlántico cobraba supremacía el reconocimiento de la actividad cognoscitiva del sujeto y la forma en que éste construye conocimientos sobre el mundo.

En el escenario de los estudios cognitivos del año 1968 aparece la obra titulada Educational Psychology: a cognitive view escrita por David Paul Ausubel, que constituye uno de los hitos fundacionales del paradigma del aprendizaje significativo, inscripto en la corriente cognoscitiva del aprendizaje y la enseñanza. En línea con este paradigma, el tema que aborda esta obra ha constituido y continúa siendo un aporte genuino a las investigaciones contemporáneas, refrendado por estudios constructivistas. Evidencias de este aporte están

Cita sugerida: Palacios, A., y Pedragosa, M. A. (2017). Introducción: pasado y presente del aprendizaje y la enseñanza significativos. Archivos de Ciencias de la Educación, 11(12), e028. https://doi.org/10.24215/23468866e028 
plasmadas en el contenido de los artículos que se presentan, ilustrados con indagaciones actuales en áreas específicas del ámbito educativo.

La publicación de este dossier reviste especial importancia para la Cátedra de Psicología Educacional de la Facultad de Humanidades y Ciencias de la Educación, Universidad Nacional de la Plata, por tres razones principales. La primera, por la posibilidad de reunir de manera inédita en nuestro ámbito académico, contribuciones que muestran la vigencia de la propuesta ausubeliana en trabajos de investigadores latinoamericanos. La segunda porque, como equipo de trabajo, nos permite recuperar y poner en valor el haber tenido el privilegio de acceder a las primeras traducciones de los trabajos de David Paul Ausubel hechas en el país y de socializarlas en nuestro ámbito académico. La tercera reside en que nos permite revitalizar nuestra participación institucional en los encuentros sobre el tema, que se vienen celebrando de manera sostenida desde el año 1992 y nuestra colaboración con la red académica internacional que sigue vigente en torno al aprendizaje significativo.

La orientación que asumen los artículos ha sido incorporada con frecuencia en las líneas de investigación que venimos desarrollando en la Cátedra desde la restauración de la democracia en nuestro país. La experiencia recogida nos ha permitido profundizar los desarrollos teóricos y reflexionar acerca de la contribución sustantiva que esta orientación puede brindar a la formación de docentes e investigadores en el campo de las Ciencias de la Educación. También la posibilidad de articular este enfoque con otras corrientes del constructivismo en el área de la Psicología Educacional. Por ello, no dudamos que este dossier será de utilidad a todos aquellos interesados en la materia, y de manera esencial a quienes se interesen por entender los procesos de enseñanza y aprendizaje escolares en una perspectiva psicopedagógica, para la construcción de una educación inclusiva.

Este dossier está compuesto de tres artículos que abordan cuestiones sobre el aprendizaje y la enseñanza con distintos énfasis. Advertidas del riesgo de simplificar merecen un comentario los temas abordados, a la luz de las trayectorias de sus autores. El primero se titula "Aprendizaje significativo como un referente para la organización de la enseñanza”, el autor es Marco Moreira Antonio, de la Universidad Federal Do Río Grande Do Sul (UFRGS), discípulo de Joseph Novak en la Universidad de Cornell, reconocido actualmente como el principal referente experto del paradigma del aprendizaje significativo. Por su amplia trayectoria en la formación de docentes, investigadores, tesistas y su vasta producción de aportes al conocimiento, le valieron reconocimientos académicos del más alto nivel, como dos doctorados honoris causa y el protagonismo más destacado en eventos mundiales sobre enseñanza de la ciencia. Con este caudal, a lo largo del artículo, Moreira ofrece un panorama de los postulados centrales de la perspectiva clásica de la teoría del aprendizaje significativo de Ausubel y, también, de propuestas más recientes en el campo de la Psicología Cognitiva, Pedagogía y Epistemología. Luego, presenta y discute una concepción original, con una mirada crítica, subversiva y antropológica de los procesos implicados en el aprendizaje.

El segundo capítulo se titula "Nuevas tecnologías de la información como facilitadoras de Aprendizaje significativo", las autoras son Irene Arriassecq y Graciela Santos. Irene Arriassecq es profesora de la Universidad Nacional del Centro de la Provincia de Buenos Aires (UNCPBA) e investigadora del Consejo Nacional de Investigaciones Científicas y Técnicas (CONICET), discípula de Marco Antonio Moreira quien dirigió su tesis doctoral en la Universidad de Burgos, titulada La enseñanza y el aprendizaje de la Teoría de la Especial de la Relatividad en el nivel medio/polimodal. Esta investigación cobra un valor singular por haberse llevado a cabo en la ciudad de Tandil, provincia de Buenos Aires y también por demostrar algunas de las principales dificultades que surgen en el proceso de enseñanza y aprendizaje en la física. Por su parte, Graciela Santos se desempeña como profesora de las Cátedras “Informática Educativa” de los Profesorados de Matemática y Física y “Enseñanza y Aprendizaje en Espacios Virtuales”, en la Universidad Nacional del Centro de la Provincia de Buenos Aires (UNCPBA). La autora investiga temas referidos a la informática 
educativa desde la Comisión de Investigaciones Científicas de la Provincia de Buenos Aires (CIC). Su área específica de trabajo aunada a la especialidad de Arriassecq redunda en una mirada innovadora sobre la potencialidad de las TIC para promover aprendizajes significativos y nuevas formas de acceso a la educación, en términos de posibilidades y recursos.

El tercer artículo se titula “El aprendizaje significativo para fomentar el pensamiento crítico” y su autor es Ricardo Chrobak, discípulo de Joseph Novak en la Cornell University. Chrobak se desempeña como profesor, investigador y director del Departamento de Física de la Facultad de Ingeniería de la Universidad Nacional del Comahue (UNCOMA). Un aspecto de singular valor es la dirección de una Maestría en Enseñanza de las Ciencias orientada hacia el aprendizaje significativo en la UNCOMA, pionera en nuestro país con esa orientación. En este artículo Chrobak recoge su amplia experiencia en la formación de docentes, investigadores y tesistas para el análisis de nuevos modelos y herramientas tendientes al desarrollo del pensamiento crítico y la creatividad.

En continuidad con esta orientación, desde 2015 el equipo de nuestra cátedra viene desarrollando un nuevo proyecto de investigación denominado Literacidad y aprendizaje: implicaciones en la formación docente, evaluación y orientación escolar. Estudios sobre las prácticas de lectura convencional y con nuevas tecnologías en escuelas públicas de la provincia de Buenos Aires, en el marco del Programa de Incentivos, Ministerio de Cultura y Educación. Este proyecto articula distintas líneas de investigación dirigidas a indagar las prácticas de literacidad escolares, en especial, de lectura de textos convencionales y electrónicos vinculados con el aprendizaje significativo. En consonancia con la propuesta de indagación de este proyecto, pretendemos reunir en los tres artículos que componen este dossier los esfuerzos por comprender mejor los espacios educativos desde la perspectiva de la significatividad, que numerosos colegas vienen trabajando en distintos ámbitos académicos. A la vez, poner de relieve la riqueza de las nuevas construcciones y fortalecer con este tipo de cooperación intelectual las propias investigaciones y aquellas que se vienen desarrollando en otras universidades de nuestro país y de América Latina.

\section{Bibliografía}

Ausubel, D. (1968). Educational psychology: a cognitive view. New York: Holt,Rinehart and Winston.

Ausubel, D., Novak, J. y Hannesian, (1988). Psicología Educativa: un punto de vista cognoscitivo. México: Trillas.

Hampson, P. y Morris, P. (1996).Understanding Cognition. Oxford:Blackwell.

Novak, J. y Gowin, D. (1988). Aprendiendo a aprender. Barcelona: Martínez Roca. 